\title{
APORTACIONES DE LA PALINOLOGÍA Y LA ARQUEOZOOLOGÍA AL ES- TUDIO DE LA MOVILIDAD DE LOS GRUPOS HUMANOS EN EL PALEOLÍ- TICO SUPERIOR EN EL VALLE GUIPUZCOANO DEL RÍO UROLA: EKAIN, ERRALLA Y AMALDA.
}

\author{
Rodrigo Portero Hernández ${ }^{1}$
}

\begin{abstract}
Resumen:
El presente artículo tiene como objetivo tratar la utilidad que los estudios de arqueobiología presentan para las investigaciones sobre la movilidad de los grupos humanos en el Paleolítico superior. Concretamente, se centra en esbozar los patrones de movilidad de los grupos cazadores-recolectores en el valle del río Urola (Gipuzkoa, Euskadi, España) durante el 19500 cal BP, a través de las correlaciones existentes entre las evidencias palinológicas y faunísticas. Las cuevas seleccionadas para este análisis son Ekain, Erralla y Amalda, situadas todas ellas en el curso medio del río Urola. Con los datos arquezoológicos y palinológicos correlacionados, se ha procedido a establecer las áreas de captación de los recursos faunísticos de los grupos humanos que habitaron el territorio a estudiar, y se han establecido las relaciones existentes entre los tres yacimientos, presentando un modelo de movilidad adecuado a las características que muestran las variables de cada una de estas cuevas.
\end{abstract}

\section{Palabras clave:}

Arqueozoología, Palinología, Movilidad, Paleolítico superior, Península Ibérica.

\section{Abstract:}

The objective of this article is to analyze the use of the studies in archaeobiology for the research about the mobility of the human groups in the Upper Palaeolithic. Specifically, it focuses on outlining the patterns of mobility of the hunter-gatherer groups who inhabited the Urola river valley (Gipuzkoa, Basque Country, Spain) during the $19500 \mathrm{cal}$ BP, using the correlations between the palynological and the faunistic evidences. For this analysis, we have selected the caves of Ekain, Erralla and Amalda, all of them located in the middle Urola River. With the zooarchaeological and palynological evidences correlated, we have proceeded to establish the catchment areas of the faunistic resources consumed by the human groups that inhabited the studied territory, and to establish the relations between the three archaeological sites, presenting a mobility model according to the characteristics of each of the caves.

\section{Key Words:}

Zooarchaeology, Palynology, Mobility, Upper Palaeolithic, Iberian Peninsula.

1Departamento de Prehistoria, Historia Antigua y Arqueología. Facultad de Geografía e Historia. Universidad de Salamanca. C/Cerrada de Serranos s/n., 37002. Salamanca. 


\section{Introducción}

La movilidad, como característica inherente al ser humano, es una realidad dinámica que debe ser estudiada por arqueólogos, prehistoriadores e historiadores en cualquier ámbito de la investigación. Para el caso del Paleolítico, la importancia de los estudios sobre movilidad radica en que los materiales descubiertos a través del registro arqueológico parecen indicar relaciones entre diferentes grupos humanos, como demuestran distintas investigaciones con relación a los utillajes líticos y óseos, al arte, a las materias primas, a las conchas marinas, etc. (ver Álvarez-Fernández, 2006; Cazals y Bracco, 2007; Rivero, 2010).

Estos datos no son los únicos que nos permiten atestiguar la movilidad de los grupos humanos en el Paleolítico superior. Los datos provenientes de la arqueobiología presentan toda una serie de elementos que permiten esbozar los patrones de movilidad de estas gentes. En este caso se utilizan los datos palinológicos y arqueozoológicos publicados de cada una de las cuevas que abarca nuestra área estudio en estrecha correlación. Estas correlaciones permiten, a través los datos palinológicos, reconstruir la cobertera vegetal a través de la cual los animales y los grupos humanos se desplazan en busca de alimento, al mismo tiempo que los datos arqueozoológicos permiten desentrañar la complejidad de las técnicas cinegéticas unidas a una base ambiental y geográfica de un determinado territorio, en este caso el valle medio del río Urola (Gipuzkoa). De esta manera, la información sobre el espacio que rodeó a los grupos paleolíticos, junto con el registro arqueológico, son los objetos de estudio que permiten reconocer los procesos sociales, económicos, culturales, etc., unidos a una base ambiental y geográfica de un determinado territorio; ya que, como señalan M.Á. Fano y O. Rivero (2012: 211), el buen conocimiento de un determinado yacimiento permite plantear hipótesis relevantes con relación a la movilidad y al modo en el que una sociedad gestiona su territorio. Para ello, es necesario contrastar hipótesis y realizar una comparativa entre los distintos sitios arqueológicos de un entorno, con el fin de caracterizar las ocupaciones y establecer un modelo para la dinámica del poblamiento.

Por lo tanto, el objetivo aquí es el esbozar los patrones de movilidad existentes entre Ekain, Erralla y Amalda dentro del marco cronológico del Paleolítico superior. Pero, ¿cómo definimos la movilidad? Podemos aceptar la definición que realizan M.Á. Fano y O. Rivero (2012: 209), quienes la entienden como una propiedad de los individuos y de los grupos sociales, los cuales tienen la capacidad de desplazarse en el espacio según sus deseos, conveniencias y posibilidades, habiendo diferentes grados y tipos de movilidad (exploratoria, logística, residencial, etc.).

En lo referente a la metodología empleada en este estudio, se ha optado por utilizar las cronologías de C14 en años BP (tanto calibradas como sin calibrar), ya 
que éstas muestran los momentos en los que estas cuevas cuentan con ocupaciones simultáneas en el territorio.

\section{Marco geografico y cronológico de estudio}

Las cuevas de Ekain, Erralla y Amalda se sitúan en el valle del río Urola (Gipuzkoa), que recorre, de sur a norte unos $59 \mathrm{~km}$ de distancia y muere en el mar Cantábrico (Figura 1).

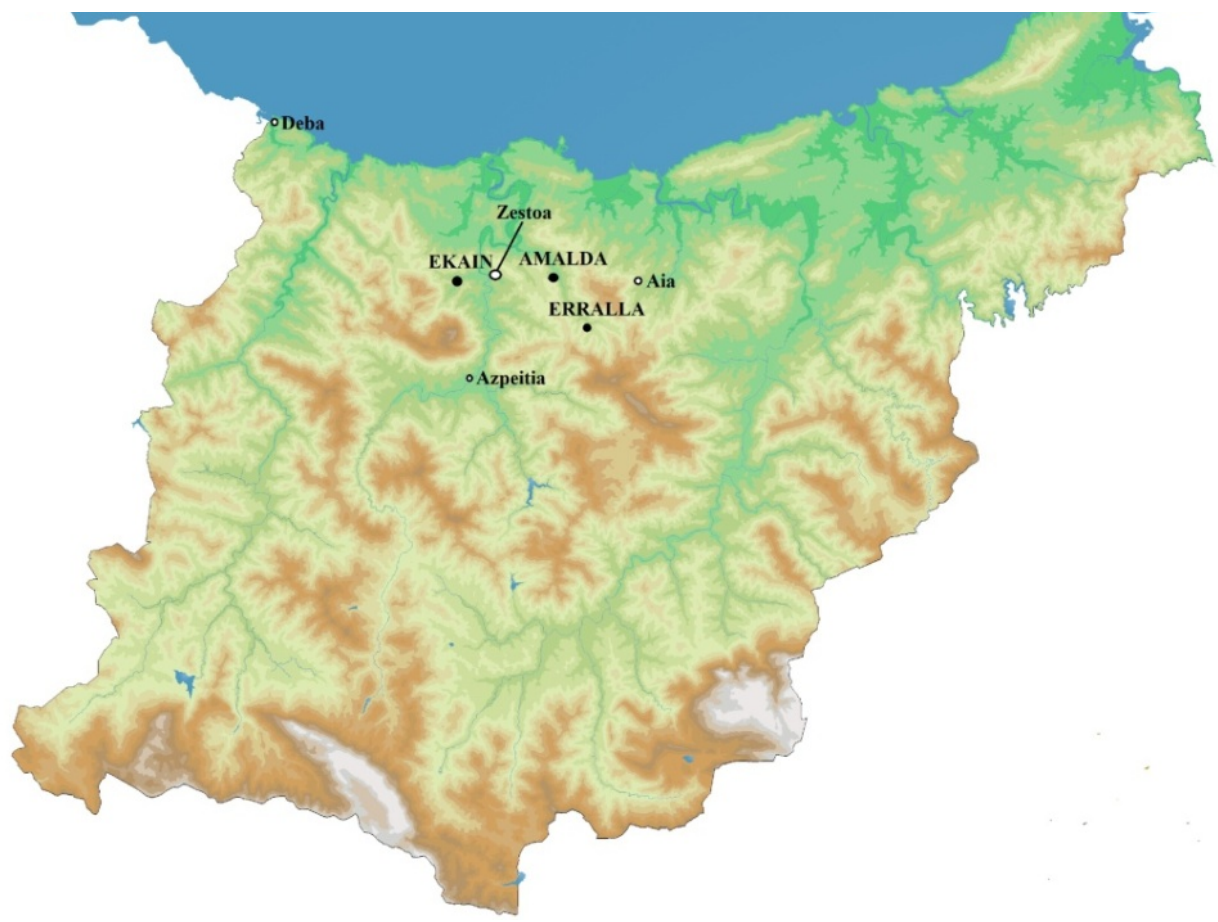

Fig 1. Mapa de la situación geográfica de Ekain, Erralla y Amalda. Fuente: Diputación Foral de Gipuzkoa-Gipuzkoako Foru Aldundia. www.gipuzkoa.eus.

\section{La cueva de Ekain (Deba, Gipuzkoa)}

La cueva de Ekain, famosa por sus pinturas rupestres, está situada en la ladera oriental de una colina que lleva su mismo nombre a 90 m.s.n.m. Cercana a ella se encuentran los regatos de Goltzibar y Beliosoerreka, los cuales se unen cerca de su boca formando el arroyo Sastarrain, que desemboca en el río Urola. En la actualidad la costa se sitúa, en línea recta, a unos $7 \mathrm{~km}$ de distancia de la cueva (Altuna, 1984a). La secuencia crono-cultural de Ekain para el periodo de estudio abarcaría el Magdaleniense Inferior (Nivel VII). 


\section{La cueva de Erralla (Zestoa, Gipuzkoa)}

La cueva de Erralla se localiza en el municipio de Zestoa, ubicada sobre el monte Ezkurruaitz, situado en la cuenca de recepción del torrente de Altzolaras, regato que desemboca en el río Urola. El entorno de la cavidad está constituido por una zona escarpada, especialmente en dirección sur y noroeste. Se encuentra a 230 m.s.n.m. y la distancia a la costa actual, en línea recta, es de unos $10 \mathrm{~km}$ (Altuna y Areso, 1985). En esta investigación se analiza la información del Magdaleniense Inferior (nivel V).

\section{La cueva de Amalda (Zestoa, Gipuzkoa)}

La cueva de Amalda está situada sobre la ladera occidental del valle de Altzolaras, a 205 m.s.n.m. Hacia el este encontramos la cumbre de Pagoeta. La distancia a la costa actual, en línea recta, es de aproximadamente 8 km (Altuna, 1990a). La presente investigación se centra en el nivel IV (adscrito al Solutrense Superior).

\begin{tabular}{|c|c|c|c|c|c|c|}
\hline $\begin{array}{l}\text { Cueva- } \\
\text { Nivel }\end{array}$ & Período & $\begin{array}{c}\text { Fecha } \pm \\
\text { desviación }\end{array}$ & $\begin{array}{l}\text { Referencia } \\
\text { laboratorio }\end{array}$ & 14C/AMS & Muestra datada & $\begin{array}{l}\text { Calibración- } \\
\text { Cal. BP }\end{array}$ \\
\hline Amalda-IV & $\begin{array}{l}\text { Solutrense } \\
\text { Superior }\end{array}$ & $\begin{array}{l}17580 \pm 440 \mathrm{BP} \\
16200 \pm 380 \mathrm{BP} \\
16090 \pm 240 \mathrm{BP}\end{array}$ & $\begin{array}{l}\text { (I-II 355) } \\
\text { (I-II 428) } \\
\text { (I-II435) }\end{array}$ & $\begin{array}{l}14 \mathrm{C} \text { conv. } \\
14 \mathrm{C} \text { conv. } \\
14 \mathrm{C} \text { conv. }\end{array}$ & $\begin{array}{l}\text { Hueso (colágeno) } \\
\text { Hueso (colágeno) } \\
\text { Hueso (Colá- } \\
\text { geno) }\end{array}$ & $\begin{array}{l}21098 \pm 617 \\
19468 \pm 499 \\
19290 \pm 350\end{array}$ \\
\hline Ekain-VII & $\begin{array}{l}\text { Magda- } \\
\text { leniense } \\
\text { Inferior }\end{array}$ & $\begin{array}{l}16510 \pm 270 \mathrm{BP} \\
16250 \pm 250 \mathrm{BP} \\
16030 \pm 240 \mathrm{BP} \\
15970 \pm 240 \mathrm{BP} \\
15400 \pm 240 \mathrm{BP}\end{array}$ & $\begin{array}{l}(\mathrm{I}-12020) \\
(\mathrm{I}-12566) \\
(\mathrm{I}-12224) \\
(\mathrm{I}-12225) \\
(\mathrm{I}-12226)\end{array}$ & $\begin{array}{l}14 \mathrm{C} \text { conv. } \\
14 \mathrm{C} \text { conv. } \\
14 \mathrm{C} \text { conv. } \\
14 \mathrm{C} \text { conv. } \\
14 \mathrm{C} \text { conv. }\end{array}$ & $\begin{array}{l}\text { Carbón } \\
\text { Carbón } \\
\text { Carbón } \\
\text { Carbón } \\
\text { Carbón }\end{array}$ & $\begin{array}{l}19793 \pm 399 \\
19462 \pm 409 \\
19213 \pm 311 \\
19156 \pm 293 \\
18516 \pm 380\end{array}$ \\
\hline Erralla-V & $\begin{array}{l}\text { Magda- } \\
\text { leniense } \\
\text { Inferior }\end{array}$ & $\begin{array}{l}15740 \pm 240 \mathrm{BP} \\
16200 \pm 240 \mathrm{BP} \\
16270 \pm 240 \mathrm{BP}\end{array}$ & $\begin{array}{l}(\mathrm{I}-12540) \\
(\mathrm{I}-12551) \\
(\mathrm{I}-12868)\end{array}$ & $\begin{array}{l}14 \mathrm{C} \text { conv. } \\
14 \mathrm{C} \text { conv. } \\
14 \mathrm{C} \text { conv. }\end{array}$ & $\begin{array}{c}\text { Hueso } \\
\text { (Colágeno) } \\
\\
\text { Hueso } \\
\text { (Colágeno) } \\
\text { Hueso } \\
\text { (Colágeno) }\end{array}$ & $\begin{array}{l}18986 \pm 287 \\
19407 \pm 390 \\
19482 \pm 408\end{array}$ \\
\hline
\end{tabular}

Tabla 1. Cuadro de las cronologías de $14 \mathrm{C}$ los niveles seleccionados de Ekain, Erralla y Amalda (a partir de Altuna, 1984, 1985, 1990a).

En lo referente al marco cronológico de estudio, se han utilizado las cronologías de ${ }^{14} \mathrm{C}$ publicadas de cada una de las cuevas (Altuna, 1984, 1985 y 1990a) 
(Tabla 1). Destacando un momento de ocupación simultáneo en el territorio en torno al $12000 \mathrm{BP}$, es decir, cerca del $19500 \mathrm{cal} \mathrm{BP}^{2}$ (Figura 2). Esto significa que en torno a esta fecha las tres cuevas situadas en el valle del Urola se encontraban ocupadas por los grupos humanos y, por lo tanto, se puede establecer un marco de movilidad coherente.

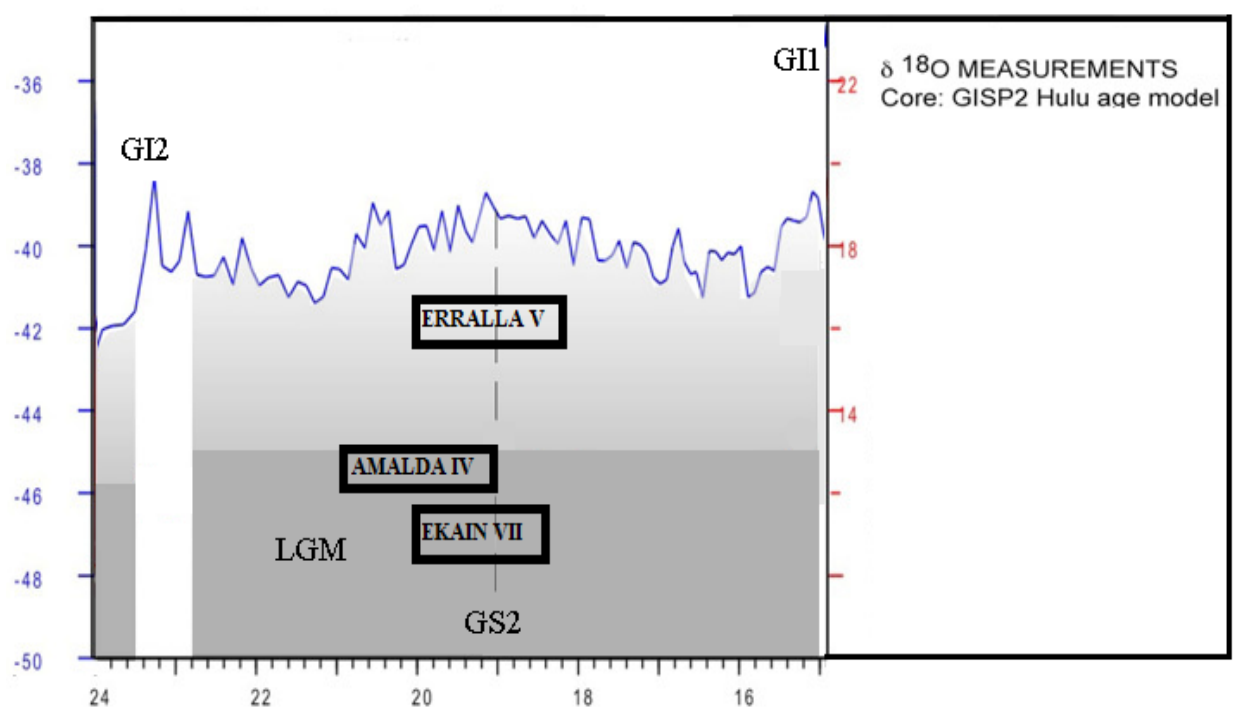

Fig. 2. Calibración de las fechas radiocarbónicas de Ekain, Erralla y Amalda a partir de: CalPal-Hulu (Weninger et al., 2012). LGM: Last Glacial Maximum. Gl1: Greenland Interestadial 1. GI2: Greenland Interestadial 2. GS2: Greenland Stadial 2.

\section{Aportaciones de la palinología}

La palinología es una ciencia dedicada al estudio de los pólenes y las esporas. La arqueología y la prehistoria se apoyan actualmente en este área de conocimiento para reconstruir, en la medida de lo posible, la flora y buscar indicadores botánicos, climáticos, ecológicos, paleontológicos, estratigráficos y económicos. Por lo tanto, permite al investigador una mejor comprensión del espacio y del territorio en los diferentes periodos de estudio, posibilitando un estudio más amplio de las condiciones ambientales en las que éstos vivieron y permitiendo el avance en el conocimiento del medio que les rodeó. La metodología que se emplea en los estudios palinológicos sigue los siguientes pasos: el muestreo, los tratamientos

2 Para la calibración de las fechas radiocarbónicas (BP) en fechas de calendario (cal BP) hemos utilizado el programa CAL PAL (Weninger et al. 2012). 
físicoquímicos, la determinación y el recuento del polen, la representación gráfica y la interpretación de los resultados (Iriarte, 2011; López, et al. 2013).

La Palinología, sin embargo, cuenta con algunas críticas y limitaciones, como la dificultad para reconocer y determinar el polen, la forma de calibrar sus porcentajes, los riesgos de contaminación y las diferentes formas de polinización posibles por causa de insectos, por trasporte eólico, etc. (ver Gómez, 1983: 29; López, et al., 2013: 284). Además, los estudios palinológicos en cuevas cuentan con otros problemas añadidos, como son las alteraciones y los desplazamientos post-deposicionales, que hacen difícil su interpretación (Sánchez, 1996), por lo que deben ser valorados antes de realizar las interpretaciones polínicas.

Para este estudio, partimos del hecho de que existe una estrecha relación entre la fauna y la flora y entre éstas y el grupo humano dentro de un plano económi$\mathrm{Co}$, ya que los animales se mueven en busca de alimento y el grupo humano se desplaza al compás de los recorridos de las manadas de estos animales. La palinología nos va a permitir, a través del estudio porcentual del polen, acercaremos al espacio vegetal del entorno paleolítico mediante la reconstrucción de su cobertera vegetal, observando los procesos de cambio en el tiempo y, posteriormente, su interacción con la fauna, el clima y los grupos de cazadores-recolectores paleolíticos que economizan el medio. Así, se puede investigar la variación de las distintas formas vegetales a lo largo de una secuencia estratigráfica con el fin de apreciar los cambios interactivos que sufren las diferentes especies, observando el aumento y la disminución, la aparición y desaparición de las especies vegetales y, posteriormente, cómo afecta su cambio a la fauna y las estrategias de movilidad de los grupos humanos.

El proceso que hemos seguido en nuestra investigación es similar al realizado por A. Gómez (1983) para la cueva de Tito Bustillo y se detalla a continuación:

1. Establecer los porcentajes de especies que se dan en las muestras polínicas de las tres cuevas, estimar la proporción de campo abierto frente al sector boscoso y observar los porcentajes de cada especie; es decir, establecer una cliserie por muestra.

2. Situar y distribuir cada especie según su mayor o menor predisposición a las condiciones geográficas, geológicas y edafológicas. Tener en cuenta, para la situación de las diferentes especies, la insolación, la altitud, etc., factores que influyen en ellas y que influirán en su situación dentro del valle.

3. Establecer el espectro biológico de las especies en el año. Esto permite conocer cuándo florece y cuándo da fruto cada una de ellas. 
Todos estos pasos se aplican al estudio de las tres cuevas en el momento cronológico escogido. El estudio de las cliseries para Ekain, Erralla y Amalda nos permite ver los aumentos y disminuciones de cada una de las especies y cotejar el entorno vegetal de alrededor de las tres cuevas (Portero, 2015). Para la situación en altura de cada una de estas especies optamos por utilizar la cartografía del Instituto Geográfico Nacional (escala 1: 25.000; hojas 63 (IV) Azkoitia y 64 (III) Villabona). Para los mapas geológicos utilizamos los expendidos por el Ente Vasco de la Energía (escala 1: 25.000; hojas 63 (IV) Azkoitia y 64 (III) Villabona). De esta manera se puede valorar el terreno geográfico y geológico a través de una línea recta que una las tres cuevas. A continuación, y con los mapas montados, nos centramos en las especies vegetales que han resultado de los análisis polínicos y les buscamos una ubicación en ellos. Para la ubicación en altura y por condiciones geológicas y edafológicas de estas especies, utilizamos los cinco paisajes de montaña establecidos E. Guinea (1953). Así, a través de esta información situamos las especies con relación a su altura y a su afinidad con los suelos, y establecemos su relación con las zonas de solana y umbría; esto es lo que se denomina "estudio de las catenas", que nos ofrece datos precisos sobre la situación de las especies sobre el terreno.

Hay que indicar, sin embargo, que las altitudes a las que se representan estas especies posiblemente varíen en épocas de intenso frío; además, el paisaje sufrió una serie de cambios en cada uno de los periodos que hay que tener muy presentes. En general, las nieves perpetuas para el Paleolítico se pueden situar sobre los 1550 m.s.n.m. (Gómez, 1981: 24).

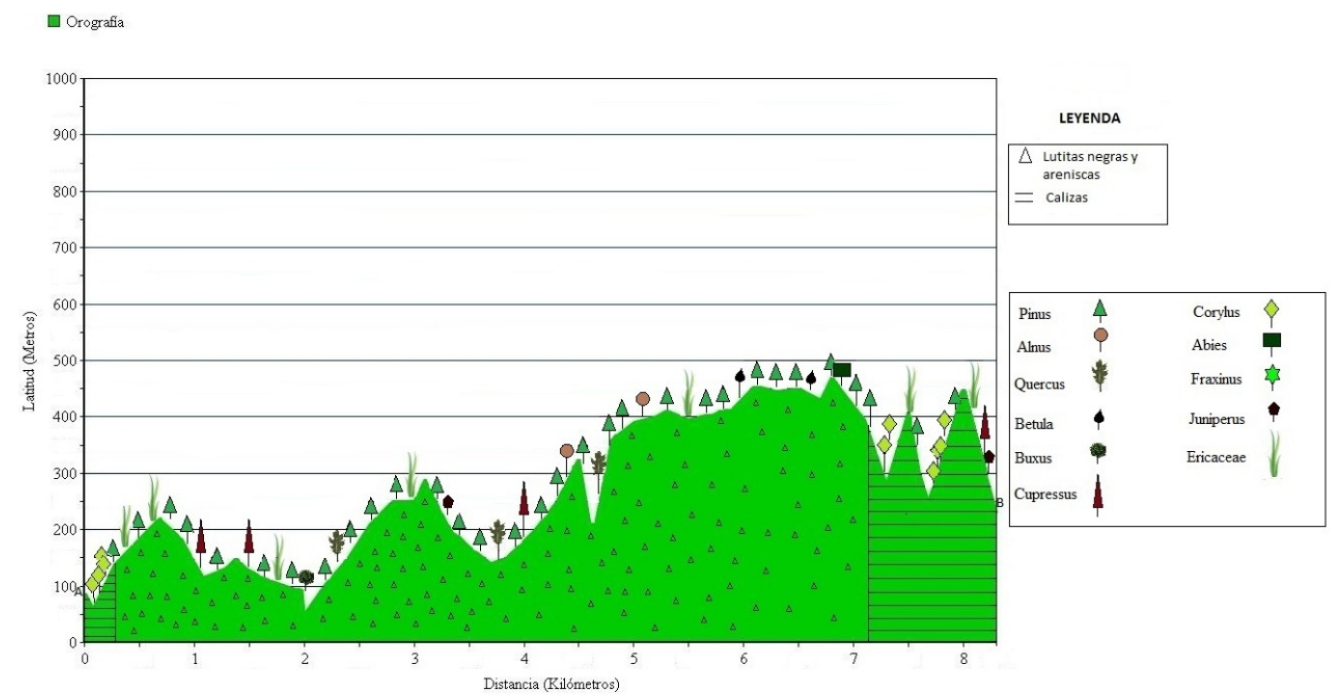

Fig. 3. Reconstrucción de la cobertera vegetal en el corte A-B ca. del 19500 cal BP. 
Antes de reconstruir la cobertera vegetal de cada uno de los cortes, quedaría establecer el espectro biológico de las especies más representativas para el estudio, es decir, establecer cuando florecen y dan fruto. Con toda esta información recopilada realizamos una reconstrucción de la cobertera vegetal paleolítica para cada uno de los momentos escogidos. Para ello utilizamos una línea recta que una las tres cuevas en su medio físico y geológico estableciendo 3 distancias: A-B (Ekain-Erralla) 8,3 km; B-C (Erralla-Amalda) 3,9 km; C-A (Amalda-Ekain) 5,6 km y situamos cada especie según lo estudiado. Con esto, reconstruimos el medio vegetal con el que interaccionan la fauna y también el grupo humano en las tres distancias representadas (Figuras 3, 4, 5). A continuación, debemos analizar la fauna para poder establecer la relación fauna-flora y grupo humano dentro del esquema de movilidad.

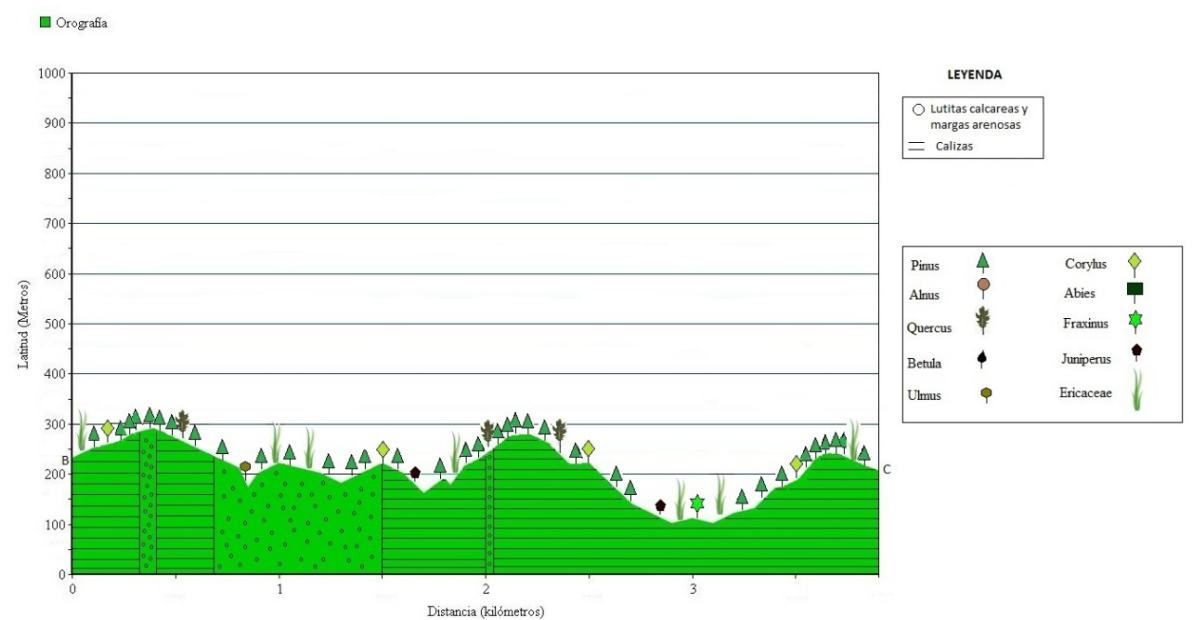

Fig. 4. Reconstrucción de la cobertera vegetal en el corte B-C ca. del 19500 cal BP.

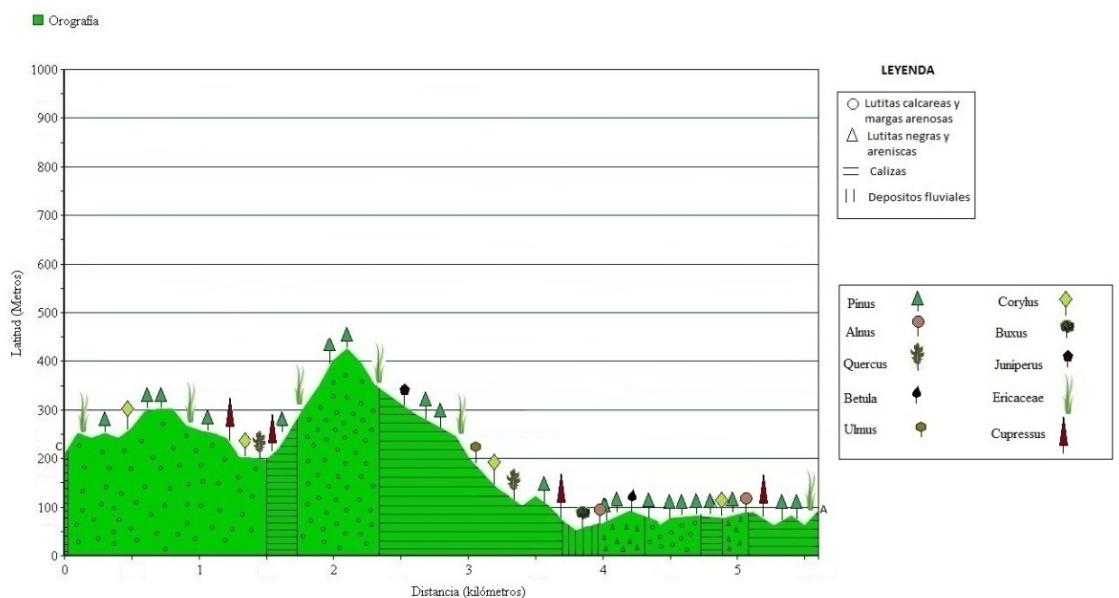

Fig. 5. Reconstrucción de la cobertera vegetal en el corte C-A ca. del 19500 cal BP. 


\section{Aportaciones de la arqueozoología}

El material arqueozoológico nos ofrece por sí mismo algunos datos de interés. El estudio de los materiales óseos de un yacimiento nos va a posibilitar un mayor conocimiento sobre la estrategia económica y el modo de captación de los recursos alimenticios de origen animal por los grupos humanos. También nos permite conocer las especies que han sido llevadas por dichos grupos al yacimiento fruto de la caza, la recolección o el carroñeo para su consumo, y las que han sido introducidas por otros agentes (aves, carnívoros, etc.).

Algunos estudios sobre fauna actual han permitido determinar la composición de la dieta de determinados animales como el ciervo a través del estudio del nitrógeno fecal (Garín et al., 2001). Con este tipo de estudios y tras haber realizado una reconstrucción de la cobertera vegetal del entorno, se puede determinar en qué cotas de altitud se encuentra una determinada especie a lo largo de todo el año. También hay estudios que han contemplado la situación de estos animales en base a sus migraciones (Gómez, 1983).

De esta forma, se puede establecer un tipo de movilidad en base a la dieta, pero también es posible determinar la ocupación de un yacimiento, es decir, su funcionalidad en relación al tipo de habitación. Esto se realiza a través de determinados marcadores faunísticos, como las aves estacionales. También por extrapolación, basándose en los periodos de nacimiento de una determinada especie, se puede establecer en qué estación del año fueron cazados y deducir la presencia humana en el yacimiento en un determinado periodo (Chaix y Méniel, 2005). El estudio de la fauna también permite documentar relaciones entre yacimientos de una misma región, distinguiendo entre lugares de consumo (campamentos base) y lugares de adquisición (cazaderos) (op. cit.: 194-195). Todo esto nos va a permitir realizar un mapa de la captación de los recursos por parte de estos grupos y determinar la duración de la ocupación de estos yacimientos.

Aplicado esto a Ekain, Erralla y Amalda en el valle del Urola, lo primero que hemos hecho ha sido un recuento del material óseo de cada uno de los niveles de estudio de todas las especies encontradas (macromamíferos, micromamíferos, malacofauna, avifauna, etc.) a través de la información faunística publicada y las consideraciones de que sobre ella realizan algunos autores (véase Altuna, 1990b; Altuna et al., 1984, 1985, 2010; Álvarez-Fernandez, 2006; Quesada, 1997, 2012; Yravedra, 2007, 2010). De esta manera, podemos determinar cuáles eran las especies más consumidas por estos grupos dentro del yacimiento y cuales fueron introducidas por otros agentes (carnívoros, aves, etc.) (Portero, 2015).

Los ungulados serían los animales cazados en estas tres cuevas, destacando la caza del ciervo en Ekain, con un 85 \% de representación sobre el total de ungulados del nivel VII. Para el caso de Erralla, en el nivel V, el ungulado más 
Portero Hernández, R.. - Aportaciones de la palinología...

consumido sería la cabra montés que alcanza casi el $90 \%$ de representatividad. El nivel IV de Amalda, sin embargo, es uno de los casos más peculiares donde el NR de ungulados parece indicar que la especie más consumida es el sarrio (63\% de representatividad), sin embargo J. Yravedra (2007) tras realizar un estudio tafonómico de los restos óseos (marcas de carnicería frente a mordeduras de carnívoro) del nivel IV, concluye que el ciervo siempre sería aportado por los grupos humanos, mientras que la cabra y el sarrio, sólo lo serían de forma esporádica, mientras que en otras ocasiones llegarían allí por otros medios. En un trabajo posterior, J. Altuna y K. Mariezkurrena (2010: 227) apuntan que la presencia de restos de rebeco en este nivel se debe fundamentalmente a la acción humana, y que si sus huesos no conservan más marcas de carnicería es a causa de la gran fragmentación del conjunto faunístico. Por otro lado, Yravedra (2010) propone, en base al amplio porcentaje de marcas de diente en los huesos de sarrio (42\%), la gran presencia de elementos axiales y la escasez de marcas de corte, que el aporte de óseo fue realizado en su gran mayoría por carnívoros de entre los cuales el leopardo podría ser el principal responsable. De este modo el ciervo y la cabra adquieren una mayor importancia en el plano de la captación de recursos.

Este tipo de observaciones se realizan sobre todas las especies representadas en cada uno de los yacimientos en estos niveles. Además, los micromamíferos, las aves y algunos ungulados, nos han permitido observar la climatología del momento a través de especies que indican climas fríos o húmedos, pudiendo correlacionarlo con las evidencias climáticas que nos indican los análisis polínicos. Para los niveles seleccionados, entre estas especies encontramos la presencia del topo nival, la perdiz nival o restos de reno (Portero, 2015). También se han tenido en cuenta aspectos como el despiece y la selección de determinadas partes anatómicas de los animales así como la recolección de determinadas especies animales por su carácter decorativo y no bromatológico. Por último, se ha podido realizar una determinación relativa de la ocupación de las cuevas por el espectro de edades que encontramos entre los individuos cazados y por el número mínimo de individuos de estas especies.

Así, para el nivel VII de Ekain, de 44 ungulados, 20 son adultos y 24 juveniles. De los 24 juveniles, 18 son cervatillos, tres son cabritos, dos son crías de sarrio y una es de caballo. De los 18 cervatillos, 16 fueron abatidos durante su primer mes de vida, siendo cazados hacia el mes de junio; los otros dos tenían tres o cuatro meses de vida, por lo que fueron abatidos también en su primer verano o a comienzos del otoño. En el caso de la cabra, sólo se ha podido determinar la muerte en un caso: un cabrito de tres ó cuatro meses, que se cazó en verano o en otoño. Todo esto lleva a J. Altuna y K. Mariezkurrena (1984: 237-238) a afirmar que el carácter de la cueva en este momento es estacional, durante los meses cálidos del año. La mayor parte de los animales (muchos, infantiles) eran traídos enteros. 
Para el nivel V de Erralla, los datos muestran como cabra fue cazada durante los meses templados del año, a excepción de uno o dos individuos, que fallecieron en invierno. Por lo que respecta al ciervo, se documentaron cinco dientes de leche y algunos fragmentos óseos de dos individuos cazados durante el primer mes de vida. En el caso de estos dos animales y teniendo en cuenta el número mínimo de individuos, la información sobre el despiece muestra cómo los cuartos traseros y delanteros son los más representados (Altuna, 1995).

En lo referente al nivel IV de Amalda, hay un importante número de individuos infantiles cazados. El ciervo y la cabra serían troceados para ser llevados al yacimiento, mientras que el sarrio sería aportado entero al yacimiento (Altuna, 1990b). En Amalda parece haber discrepancias entre una ocupación estival que se percibe a través del análisis de los ungulados del yacimiento (Altuna, 1990b; Yravedra, 2007), o invernal según los datos sobre la avifauna (Eastham, 1990).

Así, según estas consideraciones, estaríamos ante tres yacimientos de carácter estacional. Sin embargo, hay que comprobar los requisitos que cumple cada uno de ellos, ver la posible especialización y las posibilidades que pueden ofrecer, más allá de los simples datos.

\section{Patrones de movilidad en el valle del Urola}

Con todos estos datos buscamos una interrelación de la fauna con el medio vegetal por el cual se desplazan estos animales y los grupos humanos. Esto nos va a permitir comprender mejor el por qué de la elección por parte de los grupos humanos de estos emplazamientos en el valle del Urola y nos va a permitir esbozar una serie de patrones de movilidad. Así, analizando las relaciones existentes entre cada uno de los marcadores estudiados (palinológicos y faunísticos), determinamos los movimientos que realizaban estas gentes en base a la captación de recursos. Para ello buscamos un punto de unión entre la vegetación y las especies animales consumidas por los grupos humanos que nos sirva de punto de partida en la investigación.

Una de las especies más consumidas en los tres yacimientos es el ciervo. El ciervo es un animal forestal que aunque abandone el bosque para comer en el Ilano, su hábitat óptimo se encuentra en bosques mixtos mezcla de coníferas y arboles frescos. Pero si observamos la proporción del bosque en estos momentos en ninguna de las tres cuevas supera el $11 \%$ de representación frente al campo abierto, por lo que su hábitat no se encuentra muy bien representado. Buscamos ahora en la migración de este animal que nos permita ver donde se encuentra en cada momento del año. El esquema de movilidad a partir de las migraciones que propone F.F. Darling para el ciervo en la costa noroeste de Escocia lo encontramos aplicado por A. Gómez (1983: 178) en su estudio sobre Tito Bustillo. Utilizando este mismo esquema en nuestro marco de estudio, nos encontraríamos con que 
en los meses de enero a marzo los ciervos estarían en cotas bajas, desde el nivel del mar hasta los $500 \mathrm{~m}$ y, por consiguiente, se encontrarían en las tres cuevas estudiadas dado que ninguna supera esta cota de altitud (zona A). En los meses de primavera, el ciervo subiría hacia cotas más altas desde Ekain $(90 \mathrm{~m})$ hacia Amalda (205 m) y Erralla (230 m), sobrepasando esta última en busca de altitudes mayores. Durante los meses de verano el ciervo estaría en estas altitudes de más de $500 \mathrm{~m}$ (zona B). En octubre haría el recorrido inverso al efectuado en primavera y, por último, en noviembre y diciembre, estaría en las lomas bajas por debajo de los 500 m, localizándose de nuevo en todas las cuevas (Zona A) (Tabla 2).

\begin{tabular}{|c|c|c|}
\hline Enero-Febrero & $\begin{array}{c}\text { Lomas bajas: mar }-500 \mathrm{~m} . \\
\text { (Ekain, Erralla, Amalda) }\end{array}$ & $\begin{array}{c}\text { Estacional } \\
\text { Zona A }\end{array}$ \\
\hline Marzo-Abril-Mayo & $\begin{array}{c}\text { Hacia cotas más altas (Ekain } \\
\text { Erralla, Amalda) }\end{array}$ & Migración-Paso \\
\hline Junio-Julio & $+500 \mathrm{~m}$. & $\begin{array}{c}\text { Estacional } \\
\text { Zona B }\end{array}$ \\
\hline Agosto-Septiembre-Octubre & $\begin{array}{c}\text { Hacia cotas más bajas } \\
\text { (Amalda, Erralla, Ekain) }\end{array}$ & Migración-Paso \\
\hline Noviembre-Diciembre & $\begin{array}{c}\text { Lomas bajas: mar -500 m } \\
\text { (Ekain, Erralla, Amalda) }\end{array}$ & $\begin{array}{c}\text { Estacional } \\
\text { Zona A }\end{array}$ \\
\hline
\end{tabular}

Tabla 2. Esquema de movilidad de ciervo aplicado al marco de Ekain, Erralla y Amalda (a través del esquema de Gómez, 1983).

La caza de este animal, según los datos aportados por la fauna, se realizaba en los meses cálidos en los tres yacimientos, es decir, en zonas por encima de $500 \mathrm{~m}$ (zona B) y por lo tanto, en lugares alejados de las tres cuevas. En base a estas evidencias, podemos afirmar que el esquema de F.F. Darling no es válido al aplicarlo a esta zona. Las hipótesis que se pueden barajar son que, o bien estos animales fueron abatidos cerca de las cotas por encima de los $500 \mathrm{~m}$ y posteriormente transportados a los yacimientos, o bien fueron cazados en momentos de paso hacia cotas más bajas, cuando retornaban a las tres cuevas, restringiéndose si fuese así, la caza, a los meses otoñales.

La respuesta a estos interrogantes puede estar en la base de la alimentación del ciervo actual en el valle del Urola, lo cual pueda ayudar a comprender mejor la relación fauna-vegetación dentro de una geografía particular. Sin embargo, desconocemos estudios de la dieta del ciervo no sólo para nuestra área de estudio, sino también para la región cantábrica. No obstante, se ha investigado la composición 
de la dieta del ciervo en la Reserva de Caza de la Garcipollera (Huesca) basándose en el nitrógeno fecal (Garín et al., 2001). Ésta permitió mostrar los tipos de alimentos más importantes en su dieta estacional, determinando que el pino es la planta más abundante. En general, las leñosas también son una parte importante en la misma, sobre todo en los meses de otoño e invierno, cuando las gramíneas y las dicotiledóneas escasean, para ser, estas últimas, en primavera y verano, más consumidas que las leñosas. Las bellotas/hayucos serían también un complemento en la dieta, sobre todo los meses de otoño e invierno.

Esta información la hemos aplicado a nuestra zona de estudio, aun siendo conscientes de que analizamos un área geográfica diferente. Así ca. del 19500 cal. BP, las gramíneas serían el alimento principal del ciervo, seguidas por las leñosas (pino y enebro) y las dicotiledóneas, a las que habría que añadir bellotas o hayucos procedentes de robles y encinas. En los meses de primavera y desde el punto de vista nutricional, las tres cuevas ofrecen recursos favorables al ciervo, ya que, pese a no ser un hábitat boscoso, las gramíneas primaverales suplirían en gran medida las necesidades de estos animales. En verano, cuando este animal se encuentra en cotas más altas, el consumo de leñosas y gramíneas disminuye en favor de las dicotiledóneas. En esta estación destacan las ericáceas, cuya presencia es abundante en las tres cuevas. En otoño, las leñosas vuelven a adquirir gran importancia siendo la base de la alimentación del ciervo, reduciéndose en gran medida el consumo de dicotiledóneas y de gramíneas. De nuevo el pino, el enebro y el Quercus (que ahora empieza a dar su fruto), serían la base de la alimentación en esta estación. En invierno el esquema es muy parecido al anterior, pero la importancia de las leñosas es aún mayor.

Todos estos datos nos llevan a plantearnos que, pese a que el hábitat óptimo no parece estar muy representado en el bosque, según los datos obtenidos a partir de la dieta el ciervo podría encontrarse en las proximidades de las tres cuevas. Consideramos que su caza podría realizarse perfectamente a lo largo de todo el año, más en Ekain, por sus características orográficas, que en Erralla y Amalda, donde la caza se centra en los animales de roquedo.

Este proceso que aquí se presenta para el ciervo debe realizarse sobre todos aquellos recursos de origen animal consumidos por los grupos humanos. Esto es, el resto de los ungulados cazados, los recursos marinos e incluso, aunque su consumo sea menos probable, de algunas aves (Portero, 2015). También entran dentro de este análisis de movilidad aquellos recursos animales utilizados como objetos de adorno colgantes por estos grupos. Para estas tres cuevas hay que destacar, en el caso de Erralla, dos conchas perforadas (Littorina saxatilis y Nassarius reticulatus) y un tubo de Dentalium sp. Estas dos últimas citadas carecen de valor alimenticio, siendo recogidas en las playas. En Erralla también se documenta un resto de viera (Pectem maximus) que vive en aguas profundas, por lo que el fin de su recogida no fue el alimenticio. Seguramente se recogió en la playa una 
vez que el animal había muerto. En Amalda, los ejemplares perforados son tres conchas de las especies Nucella lapilus, Trivia sp. y Littorina obtusata (ÁlvarezFernández, 2006). Entre estos objetos de adorno habría que incluir una falange de halcón/rapaz perforada documentada en el nivel V de Erralla (Eastham, 1985). Desconocemos si el animal fue abatido o si este hueso se recuperó de un animal ya muerto. También podrían ser evidencias de objetos de adorno los dos huesos (ulna y metacarpo) de Tringa totanus, ya que pudieron recogerse con el fin de obtener las plumas de este animal.

De esta forma, lo que esto nos indica a propósito de la movilidad en el valle del Urola es que los movimientos de estas gentes, en base a la captación de recursos, no fueron sólo de carácter alimenticio, sino también de tipo decorativo, seleccionando determinados materiales para ello y transformándolos después en objetos de adorno.

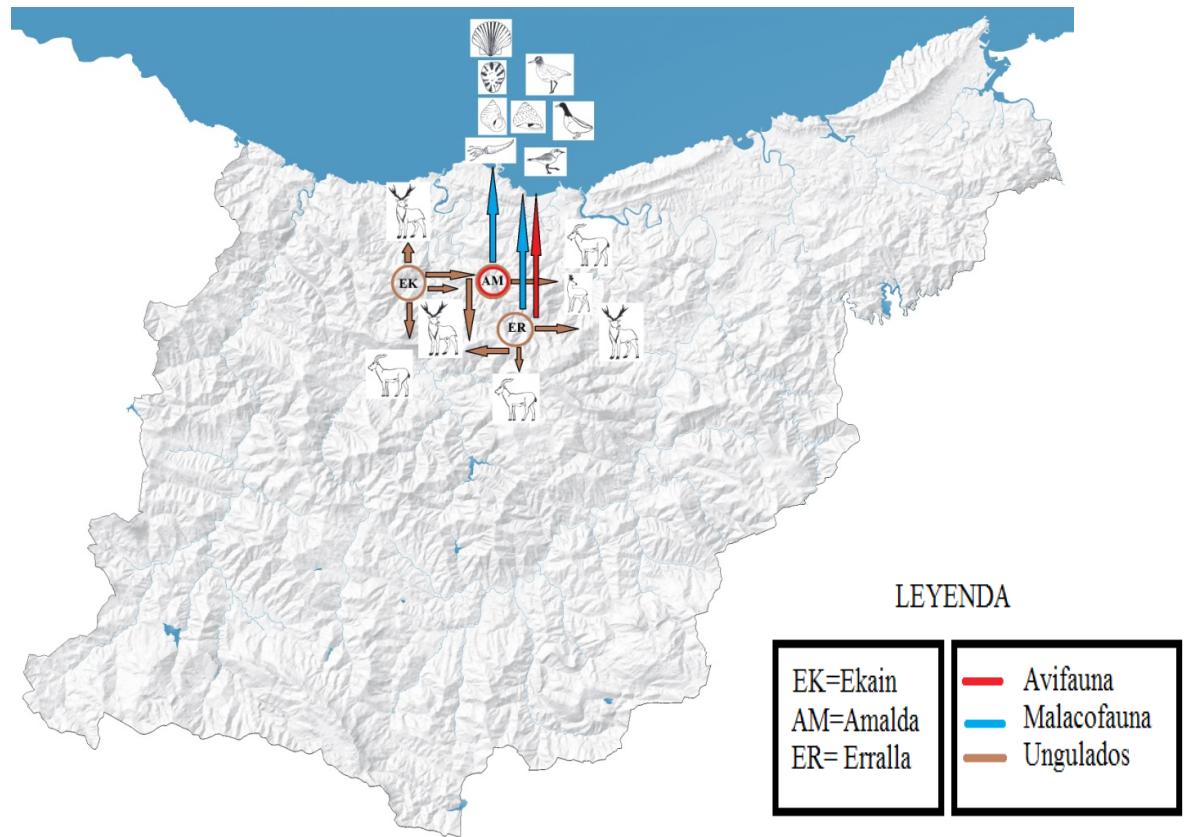

Fig .6. Mapa de captación de los recursos faunísticos ca. del 19500 cal BP. (Fuente del mapa: Diputación Foral de Gipuzkoa-Gipuzkoako Foru Aldundia. www.gipuzkoa.eus).

Con todos los datos hasta ahora correlacionados, podemos realizar un mapa de movilidad de los grupos humanos que habitaron estas cuevas en base a los recursos faunísticos y también esbozar posibles patrones de movilidad en el territorio para ca. del 19500 cal BP (Figura 6). Todos estos patrones que se trazan son los que podemos documentar a través de la información disponible, pero seguramente fueran más amplios en todo el territorio, ya que hay que contar, 
por ejemplo, con especies vegetales que podían consumirse en el ámbito de este paisaje. También hay que señalar que no existen en ninguna de las tres cuevas investigadas estudios sobre macrorrestos vegetales que podrían ofrecernos datos de interés en este sentido. Con todo ello, a través del mapa de captación de recursos y los datos que sobre la fauna y la flora poseemos, podemos esbozar los primeros patrones de movilidad para el valle del Urola.

Desde este punto de vista de la movilidad, parece acertado pensar que para ca. del 19500 cal BP desde Ekain el grupo abatía a las manadas de ciervos cuando se situaban en zonas bajas cercanas a la cueva, tras lo cual se desplazarían a Erralla en una movilidad horizontal siguiendo las manadas en su proceso migratorio hacia cotas más altas. Establecían así su estancia en Erralla y Amalda, donde la orografía ofrece otro tipo de biotipos faunísticos que eran aprovechados por estas gentes dado su menor coste de adquisición y transporte (cabra y sarrio). Allí se instalarían hasta que las manadas de ciervos invirtiesen su migración hacia cotas más bajas, retornando en estos momentos a Ekain. Esto implicaría que el grupo humano se encontraría en Ekain al menos en los meses de primavera y comienzos de verano (momento en el que abatían a las ciervas parturientas que han quedado rezagadas del grupo), desplazándose en esta última estación hacia Erralla y Amalda, donde en los meses de verano y otoño abatirían animales de roquedo. En los últimos momentos otoñales y principios de invierno, regresarían a Ekain siguiendo las manadas de ciervos.

Esto implicaría la recuperación del biotipo del ciervo en los momentos en los que el grupo se encuentra en Erralla y Amalda ya que, como señala A.B. Marín (2010: 298), el ciervo es la especie más sensible a la presión cinegética, dada su menor tasa de reemplazo, y su extinción repentina podría provocar episodios de hambruna. Se conformaría así una estrategia de caza muy elaborada que permite una mayor explotación del medio.

Por otro lado, con estos datos podemos intentar aproximarnos a la funcionalidad de los tres yacimientos estudiados en torno al $19500 \mathrm{cal} \mathrm{BP.} \mathrm{Para} \mathrm{ello} \mathrm{se}$ puede utilizar la clasificación que realiza al respecto P. Utrilla (1994). Aplicada al marco de estudio propuesto (Portero, 2015), Ekain podría ser un santuario o lugar de reunión, ya que posee manifestaciones de arte parietal, muestra una gran habitabilidad, presenta arte mobiliar con variedad de estilos, niveles estratigráficos de gran potencia, y una ocupación estacional en verano. Sin embargo, no cuenta con gran amplitud, ni gran diversidad en su utillaje. Erralla sería un campamento especializado, ya que posee un pequeño tamaño y muestra ocupación estacional, sin embargo, tiene gran variedad de utillaje lítico y óseo, en contradicción con este tipo de asentamientos. Para Amalda es difícil distinguir entre cazadero y campamento base, ya que responde a una gran habitabilidad, diversidad de utillaje y presencia de objetos-adorno, características de un campamento base. Sin embargo, y según J. Yravedra (2007), la cueva posee un carácter estacional, 
además de una mala accesibilidad, características de un cazadero estacional (esta última opción es la que nos parece más convincente). El problema de estas categorizaciones es que una cueva puede responder como hemos visto a más de un modelo, dado que no se puede resumir la complejidad de los yacimientos a través de dos variables (cazadero vs. campamentos/santuarios).

Otro tipo de evidencias que permiten afinar más en la determinación de la funcionalidad de cada uno de estos yacimientos las encontramos en las industrias líticas y óseas y las representaciones artísticas. Esto nos ofrecerá una mayor cantidad de datos a interrelacionar y permitirá crear un modelo de movilidad que se ajuste a un marco concreto. También, se puede optar por correlacionar las evidencias con modelos de movilidad ya propuestos por otros autores como $\mathrm{Vi}$ ta-Finzi y Higgs (1970), Binford (1981), Butzer (1989) o Grøn y Kuznesov (2004).

\section{Conclusión}

Tal y como se ha venido señalando a lo largo de las anteriores páginas, para valorar el proceso que aquí se presenta queda claro que la palinología y la arqueozoología son dos disciplinas que nos ofrecen toda una serie de datos a interrelacionar, necesarios en el ámbito de la investigación de la movilidad en el Paleolítico superior. Hemos tratado de esbozar la forma en que cada una de estas disciplinas nos aporta su grano de arena en la reconstrucción de las formas de vida de estas gentes permitiéndonos, a través de la estrecha correlación entre ambas, percibir los procesos económicos y sociales que hacen que estas gentes se desplacen por el territorio. Todo ello a través de una escala de análisis reducida (el valle del Urola).

A través de la palinología se han observado las variaciones vegetales a largo de la secuencia estratigráfica de estudio y se ha podido reconstruir la cobertera vegetal del entorno paleolítico en un momento concreto del Paleolítico superior (19500 cal BP). Este es el tapiz vegetal por el que se desplazan los animales y los grupos humanos. En el caso de la arqueozoología, se ha observado cuáles eran las especies cazadas y recolectadas por estos grupos a través de una serie de marcadores, lo cual ha permitido correlacionar las evidencias climáticas de fauna fría con la flora observada en los análisis polínicos; además, se han fijado los movimientos de estos animales (en este caso el ciervo) a través del valle estudiado, y se ha creado un mapa de la captación de recursos de origen animal por los grupos cazadores-recolectores.

A partir de las aportaciones realizadas por cada una de estas disciplinas y la correlación entre ambas, se han esbozado una serie de patrones de movilidad entre las tres cuevas vascas que han sido señaladas a lo largo de estas páginas.

Como apunte final cabría decir que este tipo de análisis, que resultan muy útiles para esbozar estos patrones de movilidad dentro de un marco reducido, 
nunca deben tomarse de manera directa y sin ser correlacionados con todas las evidencias posibles que puedan extraerse sobre los yacimientos y será necesario un esfuerzo transdisciplinar para poder llegar a obtener un mayor conocimiento sobre el Pasado de nuestra especie.

\section{Bibliografía}

J. Altuna: "Situación de la cueva en su ámbito geográfico. Relación con otros yacimientos prehistóricos del entorno". En J. Altuna, y J.M. Merino (eds.): El yacimiento prehistórico de la cueva de Ekain (Deba, Guipúzcoa). Eusko Ikaskuntza, San Sebastián: 1984a, 9-15.

—(1984b): "Historia de las excavaciones. Descripción del yacimiento. Resumen estratigráfico del relleno. Utilización del espacio. Dataciones absolutas". En J. Altuna y J.M. Merino (eds.): El yacimiento prehistórico de la cueva de Ekain (Deba, Guipúzcoa). Eusko Ikaskuntza, San Sebastián, 17-45.

- (1985): "Dataciones del C14. Comparación con las dataciones de otros yacimientos". Munibe, 37, 25-28.

—(1990a): "Situación y descripción de la cueva de Amalda. Historia de las excavaciones. Descripción del relleno. Estructuras en el yacimiento. Dataciones de radiocarbono. Otros yacimientos del valle". En J. AltunA et al. (eds.): La cueva de Amalda (Zestoa, País Vasco): ocupaciones paleolíticas y postpaleolíticas. Eusko Ikaskuntza, Donostia. 9-31.

- (1990b): "Caza y alimentación procedente de Macromamíferos durante el Paleolítico de Amalda". En J. Altuna et al. (eds.): La cueva de Amalda (Zestoa, País Vasco): ocupaciones paleolíticas y postpaleolíticas. Eusko lkaskuntza, Donostia, 149-192.

J. Altuna y P. Areso: "Situación y descripción de la cueva. Historia de las excavaciones. Descripción macroscópica del relleno". Munibe, 37 (1985), 11-23.

J. Altuna, A. Baldeón y K. Mariezkurrena: Cazadores Magdalenienses de la Cueva de Erralla. Munibe, 37 (1985).

—(1990): La cueva de Amalda (Zestoa, País Vasco): ocupaciones paleolíticas y postpaleolíticas. Eusko Ikaskuntza, Donostia.

J. Altuna y K. Mariezkurrena: "Bases de subsistencia de origen animal en el yacimiento de Ekain". En J. Altuna y J.M. Merino (eds.): El yacimiento prehistórico de la cueva de Ekain (Deba, Guipúzcoa). Eusko Ikaskuntza, San Sebastián: 1984, 211-280.

—(1985): "Bases de subsistencia de los pobladores de Erralla: Macromamíferos". Munibe, 37, 87-117.

—(2010): "Tafocenosis en yacimientos del País Vasco con predominio de grandes carnívoros: consideraciones sobre el yacimiento de Amalda". En E. BAQUEDANO 
Portero Hernández, R.. - Aportaciones de la palinología...

y J. Rosell (eds.): Actas de la primera reunión de científicos sobre cubiles de hiena y otros grandes carnívoros, en los Yacimientos Arqueológicos de la Península Ibérica. Museo Arqueológico Regional (Zona Arqueológica, 13), Madrid, 214-228.

E. Álvarez-Fernández: Los objetos de adorno-colgantes del Paleolítico superior y del Mesolítico en la Cornisa Cantábrica y en el Valle del Ebro: una visión europea. Universidad de Salamanca (colección Vítor 195), Salamanca.

L.R. BINFORD: "Willow smoke and dog's tails: hunter-gatherer settlement systems and archaeological site formation". American Antiquity, 1, 45 (1980), 4-20.

K.W. Butzer: Arqueología - una ecología del hombre - . Método y teoría para un enfoque contextual. Bellaterra, Barcelona: 1989.

N. Cazals y J.P. Bracco: "Quelles relations de part et d'autre des Pyrénées durant le Magdalénien?" En N. CazAls et al. (eds.): Fronteras naturales y fronteras culturales en los Pirineos prehistóricos. Universidad de Cantabria, Santander: 2007, 125-142.

L. Chaix y P. Méniel: Manual de Arqueozoología. Ariel Prehistoria, Barcelona: 2005.

M. Dupré: "Palinología de los niveles VII a II". En J. Altuna y J.M. Merino (eds.): El yacimiento prehistórico de Ekain (Deba, Guipuzcoa). Eusko Ikaskuntza, Donostia: 1984, 61-64.

—(1990): "Análisis polínico de la cueva de Amalda". En J. Altuna et al. (eds.): La cueva de Amalda (Zestoa, País vasco) ocupaciones paleolíticas y postpaleolíticas. Eusko Ikaskuntza, Donostia, 49-52.

A. EAstham: "The Bird bones in the Cave of Amalda". En J. Altuna et al. (eds.): La cueva de Amalda (Zestoa, País vasco) ocupaciones paleolíticas y postpaleolíticas. Eusko Ikaskuntza, Donostia: 1990, 239-259.

M.Á. FANO Y O. Rivero: "El territorio y la movilidad de los cazadores del final del Paleolítico: algunas reflexiones metodológicas". En P. ARIAS et al. (eds.): El Paleolítico Superior Cantábrico: actas de la Primera Mesa Redonda, San Román de Candamo (Asturias), 26-28 de abril de 2007. Universidad de Cantabria, Santander: 2012, 207-216.

C. Gamble: Las sociedades paleolíticas de Europa. Ariel Prehistoria, Barcelona: 2001.

I. Garín, A. Aldezzabal, R. García-González, J.R. Aimartza: "Composición y calidad de la dieta del ciervo (Cervus elaphus L.) en el norte de la Península Ibérica". Animal Biodiversity and Conservation, 1, 24 (2001), 1-11.

A. Gómez: "El espacio paleolítico: Cueva Morín". Zephyrus, 32-33 (1981), 17-39

-(1983): Formas económicas del Paleolítico superior Cantábrico. Fasc.1. Tito Bustillo. Universidad de Salamanca, Salamanca. 
O. Grøn y O. Kuznetsov: "What is a hunter-gatherer settlement? An ethno-archaeological and interdisciplinary approach". En P. CROMBÉ (ed.): Le Mésolithique: Actes du XIVème Congrès UISPP, Université de Liège, Belgique, 2-8 september 2001. BAR International Series 1302, Lieja: 2004, 47-53.

E. GuineA: Geografía botánica de Santander. Diputación provincial de Santander, Santander: 1953.

M.J. IRIARTE: "Polen y vegetación en la secuencia estratigráfica de Santimamiñe (Kortezubi, Bizkaia)". En J.C. LóPEZ-Quintana (dir.): La Cueva de Santimamiñe: revisión y actualización (2004-2006). Bizkaiko Foru Aldundia-Diputación Foral de Bizkaia (Kobie, 1), Bilbao: 2011, 321-341.

J.A. Lopez-Sáez; M.J. Iriarte; F. Burjachs: "Arqueopalinología". En M. García-Diez y L. ZAPATA (eds.): Metodos y técnicas de análisis y estudio en arqueología prehistórica. De lo técnico a la reconstrucción de los grupos humanos. Universidad del País Vasco, Bilbao: 2013, 273-289.

A.B. MARín: Arqueozoología en el Cantábrico Oriental durante la transición Pleistoceno/Holoceno. La cueva del Mirón. Universidad de Cantabria, Santander: 2010.

R. Portero: La movilidad de los grupos humanos en el valle del río Urola a finales del Paleolítico superior (ca. del 19500 y del 14000 cal BP): Ekain, Erralla y Amalda. ArtGerust, Madrid: 2015.

J.M. QuesadA: "La caza en el Solutrense Cantábrico: una nueva perspectiva", Zephyrus, 50, (1997), 3-36.

O. Rivero: La movilidad de los grupos humanos del Magdaleniense en la Región Cantábrica y los Pirineos: una visión a través del arte. Universidad de Salamanca, Salamanca: 2010, (Tesis Doctoral Inédita).

M.F. SánCHEZ: "Les changements climatiques du Paléolithique Supérieur. Enquête sur le rapport entre paléoclimatologie et Préhistoire". Zephyrus, 49 (1996), 3-36.

P. UtRILla: "Campamentos-base, cazaderos y santuarios. Algunos ejemplos del Paleolítico penínsular". En J.A. LASHeRAS (ed.): Homenaje al Dr. Joaquín González Echegaray. Monografías del Centro de Investigación y Museo de Altamira, Ministerio de Cultura, Madrid: 1994, 97-113.

C. VITA-FINZI y E.S. HigGS: "Prehistoric economy in Mountain Carmel area of Palestine: site catchment analysis". Proceedings of Prehistoric Society, 36 (1970), 1-37.

B. Weninger; O. Jöris; U. Danzeglowe: Cologne Radiocarbon Calibration \& Paleoclimate Research Package. University of Cologne, Cologne: 2012.

J. YRAVEDRA: "Nuevas contribuciones al comportamiento cinegético de la Cueva de Amalda". Munibe, 58 (2007), 43-88.

-(2010): "Tafonomía en la cueva de Amalda: la intervención de carnívoros. En E. Baquedano y J. Rosell (eds.): Actas de la primera reunión de científicos sobre 
Portero Hernández, R.. - Aportaciones de la palinología...

cubiles de hiena y otros grandes carnívoros, en los Yacimientos Arqueológicos de la Península Ibérica. Museo Arqueológico Regional (Zona Arqueológica, 13), Madrid: 2010, 175-184. 